\title{
The Objective Image Quality augmentation of Noisy Images with Fuzzy C Means based JPEG Compression
}

\author{
Vanitha Kakollu \\ Research Scholar \\ Department of CSE, \\ JNTU Hyderabad, India
}

\author{
G. Narasimha \\ Department of CSE, \\ JNTUH College of \\ Engineering, \\ Sultanpur, Sangareddy, INDIA
}

\author{
P. Chandrasekhar Reddy \\ Department of Electronics and \\ Communications Engineering, \\ JNTU Hyderabad, INDIA
}

\begin{abstract}
In the epoch of Information Technology, encroachments in communication technology, computers are churning the presentation of new applications which uses images widely. As a result, the facility to accumulate and convey the image, video information in an competent manner has become very critical. In this paper, an innovative JPEG compression algorithm with Fuzzy $\mathrm{C}$ means based clustering discussed. The projected algorithm is estimated to fabricate improved results in terms of MSE, PSNR and number of bits transmitted, when judge against to the customary algorithms. The proposed JPEG algorithm augments the speed and condenses the number of encoded bits, thereby reducing the amount of memory required. The proposed approach is applied on images corrupted with Gaussian noise, Speckle noise, Poisson noise and Salt \& Pepper noise.
\end{abstract}

\section{Keywords}

Image Compression, clustering, PSNR, MSE, AD, SC.

\section{INTRODUCTION}

The two dimensional image $f(x, y)$ is given to an encoder which generates a set of symbols. We then transmit this through a channel and the encoded image is now given to the decoder. A reconstructed image $\mathrm{f}^{\prime}(\mathrm{x}, \mathrm{y})$ is generated by the decoder. The output $\mathrm{f}^{\prime}(\mathrm{x}, \mathrm{y})$ is an accurate imitation of $\mathrm{f}(\mathrm{x}, \mathrm{y})$ in lossless compression. Else it means that there is some misconception present in the re-enacted image [1]. The JPEG (Joint Photographic Experts Group), the committee that shaped the JPEG standard, is an identifiable lossy compression proposal. Not just using less memory, but also the data in the regenerated image in a JPEG compression appears very much identical. Though the quality is reduced with JPEG compression, the image will look nearly as identical as the original image. The JPEG algorithm wipes out high frequency components that the human eye can't identify.

\subsection{JPEG Algorithm}

The comparatively straightforward algorithm behind JPEG can be acknowledged through the following steps:

1. The acquired image can be divided it into 8- pixel by 8 pixel blocks. If the image size is not exactly multiplied by 8 , then add zeros in empty pixels around the edges.

2. For each 8-by-8 block, get image data such that you have values to represent the color at each pixel.

3. The Discrete Cosine Transform (DCT) of each 8-by-8 block be obtained.

4. In order to make a number of values as zero from the DCT matrix, the DCT of each 8 X8 block should be multiplied by a normalized mask.
5. Normalization abandons most of the high frequency components. Next the assortment of important 2-D normalized DCT Coefficients by traversing in ZIGZAG fashion and categorizing them in a 1-D array. In 1-D array, the two types of DCT coefficients the first one is termed as direct current (DC) element, while other coefficients are called alternating current (AC) elements. Variable length Huffman coding is used to code AC components.

6. The reverse operation of compression is decompression. First calculates the normalized DCT values by decoding the compressed bit stream by Huffman codes. Then, all DCT values are organized in 2-D array by zigzag fashion explained previously. The decoded DCT values are obtained by multiplying them with normalization coefficients. Now an IDCT is executed on the denormalized DCT array. The decoding process engenders ensuing image block will not be identical as respective original image block used during encoding[1].

\section{INTENDED INNOVATIVE JPEG COMPRESSION ALGORITHMS}

If in $8 \times 8$ blocks include lot of distinction in pixel values then the number of constructive DCT coefficients will grow to be more. Otherwise only first few DCT coefficients will be more noteworthy while others are zeros. On the application of filters the image gets smoothened as a result the distinction of the pixel values of a block abridged.

The intended JPEG algorithms are implemented in two dissimilar ways.

1) Earlier than separating the image into $8 \times 8$ blocks the images infected with Poisson, Speckle, Salt \& Pepper noise and Guassian noise is convoluted with Fuzzy C Means clustering.

2) Before the application of normalized matrix the image is convoluted with Fuzzy C Means clustering.

This paper observes the comparison between the proposed approach with the standard JPEG compression. The planned approaches illustrate enhanced results compared to the JPEG in terms encoded bits. This paper makes use of MATLAB tools to admittance the proposed algorithms and the images are downloaded from SIPI image database.

Algorithm1: Fuzzy C Means Based JPEG algorithm on noisy images.

Step1: Read the image.

Step 2: Apply speckle / Poisson/ Gaussian/ Salt \& Pepper Noise.

Step 3: Apply Fuzzy C Means clustering. 
Step 4: Standard Jpeg Compression [8, 9]

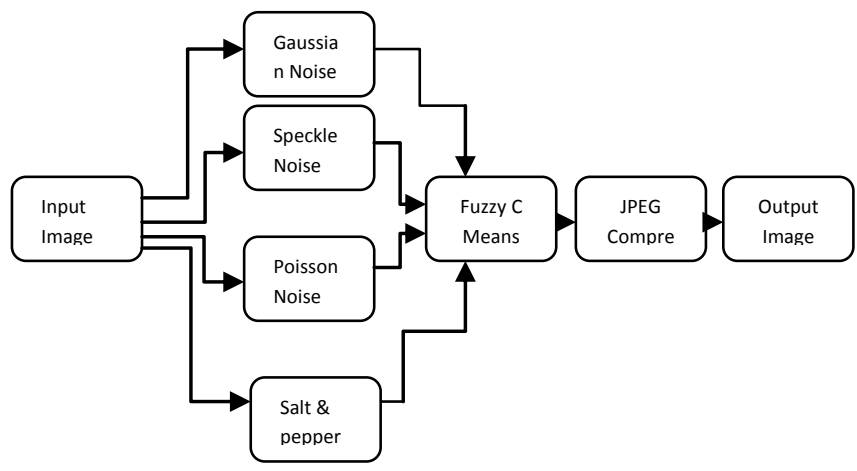

Fig1: Structure of Planned JPEG algorithms on images corrupted with various types of noise.

\section{IMPLEMENTATION OF PLANNED JPEG ALGORITHMS}

In this paper Fuzzy c means based Jpeg compression is executed on images of corrupted with different types of noises and of dissimilar sizes. Deliberation of results assigns that the newly expected compression techniques are enormously a prominent alternate since they are proved to be better in terms of image quality metrics like PSNR, MSE, AD, SC, Compression ratio.

The re-enacted image is identical to the original image with lossless compression algorithms as they not only mop out redundancy but also exterminates the redundancy present in the data they even sentinel all the information that is present in the input image.

In case of lossy compression algorithms, the output image and the input image will not be alike. So elevated compression can be achieved. We can either use subjective fidelity criteria or objective fidelity criteria for comparing the original and reprocessed image. An example for objective fidelity criteria is Peak signal to noise ratio.

Measurement of the image quality is an essential insinuation in image processing. In many of the image processing applications, assessment is coercion for the fineness of the image. The judgment of quality of the image by human is not ample. Therefore some more metrics like PSNR (Peak Signal to Noise Ratio) and MSE (Mean Square Error) are needed. PSNR is the most expert image quality metric. The differences between the restructured image and the input image will be small when the MSE value is low and PSNR value is high.

This paper spots the comparison between the proposed (Mean / Median) / Harmonic Mean / Contra Harmonic Mean based approaches with the standard JPEG compression. The deliberated approaches embody enhanced results compared to the JPEG. Out of these proposed smoothening based JPEG compressions the Harmonic Mean filter on images corrupted with on speckle noise in algorithm1, Median filter on images corrupted with salt and pepper noise in algorithm 2 and Contra Harmonic Mean filter on images corrupted with speckle noise encodes the images with fewer number of bits as a result the images will be convey with a high speed. The crucial implication in image processing is the measurement of image quality. Evaluation and assessing is obligatory for image quality in numerous image processing implementations. The refinement of human to boost the image quality is not adequate. So we necessitate some additional image quality metrics like Mean Square Error (MSE), Peak Signal to Noise
Ratio (PSNR). The number of encoded bits required to characterize the compressed image is minimized with the Harmonic Mean filter on images corrupted with Poisson noise inalgorithm1, Median filter on images corrupted with salt and pepper noise in algorithm2, and Contra Harmonic Mean filter on images corrupted with speckle noise with algorithm3 results high compression ratio than the standard JPEG compression

\section{RESULTS}

This paper presents the appraisal between the proposed Fuzzy c means based JPEG approaches with the standard JPEG compression. The wished-for approaches typify enhanced results compared to the JPEG. The proposed approach in the paper makes use of MATLAB tools for processing the images downloaded from SIPI image database.

This paper spots the comparison between the proposed Fuzzy C Means based approach with the standard JPEG compression. The premeditated approach embodies superior results compared to the JPEG. The proposed JPEG compression produces better results when the images corrupted with Speckle noise. The Proposed approach on images corrupted with speckle noise encodes the images with less number of bits as a result the images will be communicated with a elevated speed. The central insinuation in image processing is the amount of image quality. Estimate and assessing is requisite for image quality in plentiful image processing implementations. The alteration of human to enhance the image quality is not ample. So we demand some additional image quality metrics like Mean Square Error (MSE), Peak Signal to Noise Ratio (PSNR). The number of encoded bits required to exemplify the compressed image is minimized with the proposed approach on images corrupted with Speckle noise.

Table 1: Fuzzy C Means JPEG Compression on Images corrupted with Gaussian noise of size 256X 256

\begin{tabular}{|l|l|l|l|l|}
\hline Images & 5.1 .09 & 5.1 .11 & 5.1 .12 & 5.1 .13 \\
\hline $\begin{array}{l}\text { No Of Bits } \\
\text { Required }\end{array}$ & 52347 & 51910 & 58464 & 73712 \\
\hline Saved bits & 471941 & 472378 & 465824 & 450576 \\
\hline RMS Error & 2.62 & 2.54 & 2.63 & 3.55 \\
\hline Compression ratio & 10.01 & 10.099 & 8.96 & 7.11 \\
\hline PSNR & 39.80 & 40.07 & 39.77 & 37.15 \\
\hline MSE & 6.87 & 6.44 & 6.91 & 12.62 \\
\hline
\end{tabular}

Table 2: Fuzzy C Means JPEG Compression on Images corrupted with Salt \& Pepper noise of size 256×256

\begin{tabular}{|l|l|l|l|l|}
\hline Images & 5.1 .09 & 5.1 .11 & 5.1 .12 & 5.1 .13 \\
\hline $\begin{array}{l}\text { No of Bits } \\
\text { Required }\end{array}$ & 57483 & 59452 & 63046 & 70200 \\
\hline Saved bits & 466805 & 464836 & 461242 & 454088 \\
\hline RMS Error & 2.75 & 2.26 & 2.43 & 2.94 \\
\hline $\begin{array}{l}\text { Compression } \\
\text { ratio }\end{array}$ & 9.12 & 8.81 & 8.316 & 7.46 \\
\hline PSNR & 39.38 & 41.07 & 40.44 & 38.81 \\
\hline MSE & 7.56 & 5.12 & 5.92 & 8.61 \\
\hline
\end{tabular}


Table 3: Fuzzy C Means JPEG Compression on Images corrupted with Poisson noise of size 256 X 256

\begin{tabular}{|l|l|l|l|l|}
\hline Images & 5.1 .09 & 5.1 .11 & 5.1 .12 & 5.1 .13 \\
\hline $\begin{array}{l}\text { No Of Bits } \\
\text { Required }\end{array}$ & 48021 & 49567 & 55593 & 65483 \\
\hline Saved bits & 476267 & 474721 & 468695 & 458805 \\
\hline RMS Error & 2.48 & 1.9 & 2.05 & 2.87 \\
\hline $\begin{array}{l}\text { Compressio } \\
\text { n ratio }\end{array}$ & 10.91 & 10.57 & 9.43 & 8.00 \\
\hline PSNR & 40.28 & 42.61 & 41.92 & 39.00 \\
\hline MSE & 6.14 & 3.6 & 4.21 & 8.25 \\
\hline
\end{tabular}

Table 4: Fuzzy C Means JPEG Compression on Images corrupted with Speckle noise of size 256X256

\begin{tabular}{|l|l|l|l|l|}
\hline Images & 5.1 .09 & 5.1 .11 & 5.1 .12 & 5.1 .13 \\
\hline $\begin{array}{l}\text { No Of Bits } \\
\text { Required }\end{array}$ & 49323 & 54978 & 60509 & 69268 \\
\hline Saved bits & 474965 & 469310 & 463779 & 455020 \\
\hline RMS Error & 2.36 & 2.03 & 2.23 & 2.89 \\
\hline $\begin{array}{l}\text { Compressio } \\
\text { n ratio }\end{array}$ & 10.62 & 9.53 & 8.66 & 7.569 \\
\hline PSNR & 40.77 & 42.00 & 41.21 & 38.94 \\
\hline MSE & 5.49 & 4.13 & 4.95 & 8.37 \\
\hline
\end{tabular}

Table 5: JPEG Compression on Images corrupted with Gaussian noise of size 256X256

\begin{tabular}{|l|l|l|l|l|}
\hline Images & 5.1 .09 & 5.1 .11 & 5.1 .12 & 5.1 .13 \\
\hline $\begin{array}{l}\text { No Of Bits } \\
\text { Required }\end{array}$ & 131762 & 128052 & 130105 & 112493 \\
\hline Saved bits & 392526 & 396236 & 394183 & 411795 \\
\hline RMS Error & 8.30 & 8.21 & 8.14 & 7.18 \\
\hline $\begin{array}{l}\text { Compressio } \\
\text { n ratio }\end{array}$ & 3.97 & 4.09 & 4.02 & 4.66 \\
\hline PSNR & 29.79 & 29.88 & 29.95 & 31.04 \\
\hline MSE & 68.84 & 67.39 & 66.25 & 51.56 \\
\hline
\end{tabular}

Table 6:JPEG Compression on Images corrupted with Speckle noise of size 256X256

\begin{tabular}{|l|l|l|l|l|}
\hline Images & 5.1 .09 & 5.1 .11 & 5.1 .12 & 5.1 .13 \\
\hline $\begin{array}{l}\text { No Of Bits } \\
\text { Required }\end{array}$ & 85872 & 107713 & 110033 & 107065 \\
\hline Saved bits & 438416 & 416575 & 414255 & 417223 \\
\hline RMS Error & 7.07 & 7.81 & 7.65 & 6.96 \\
\hline $\begin{array}{l}\text { Compressi } \\
\text { on ratio }\end{array}$ & 6.10 & 4.86 & 4.76 & 4.89 \\
\hline PSNR & 31.18 & 30.32 & 30.49 & 31.31 \\
\hline MSE & 49.99 & 60.93 & 58.54 & 48.46 \\
\hline
\end{tabular}

Table 7: JPEG Compression on Images corrupted with Poisson noise of size 256X256

\begin{tabular}{|l|l|l|l|l|}
\hline Images & 5.1 .09 & 5.1 .11 & 5.1 .12 & 5.1 .13 \\
\hline $\begin{array}{l}\text { No Of Bits } \\
\text { Required }\end{array}$ & 81211 & 86149 & 91099 & 93492 \\
\hline Saved bits & 443077 & 438139 & 433189 & 430796 \\
\hline RMS Error & 6.71 & 6.92 & 6.94 & 5.68 \\
\hline $\begin{array}{l}\text { Compressi } \\
\text { on ratio }\end{array}$ & 6.45 & 6.08 & 5.75 & 5.60 \\
\hline PSNR & 31.63 & 31.36 & 31.34 & 33.08 \\
\hline MSE & 45.08 & 47.92 & 48.19 & 32.26 \\
\hline
\end{tabular}

Table 8: JPEG Compression on Images corrupted with Salt \& Pepper of size 256X256

\begin{tabular}{|l|l|l|l|l|}
\hline Images & 5.1 .09 & 5.1 .11 & 5.1 .12 & 5.1 .13 \\
\hline $\begin{array}{l}\text { No Of Bits } \\
\text { Required }\end{array}$ & 100843 & 97834 & 102094 & 118128 \\
\hline Saved bits & 423445 & 426454 & 422194 & 406160 \\
\hline RMS Error & 6.65 & 5.95 & 5.98 & 5.85 \\
\hline $\begin{array}{l}\text { Compressi } \\
\text { on ratio }\end{array}$ & 5.19 & 5.35 & 5.13 & 4.43 \\
\hline PSNR & 31.7 & 32.68 & 32.64 & 32.82 \\
\hline MSE & 44.27 & 35.35 & 35.71 & 34.22 \\
\hline
\end{tabular}

\section{CONCLUSION}

In this paper Fuzzy C means based JPEG compression algorithm is projected, and this algorithm is evaluated with standard jpeg algorithm on images corrupted with Gaussian, Speckle, Poisson and Salt \& Pepper noise. As in this algorithm the images can be compressed with less encoded bits as a result the loading and storing of images take less time. As MSE of the proposed approach is low compared to regular jpeg and the perfect classification correctness is augmented with the expected approach because of PSNR. The projected compression ratio can be realized with good quality image with necessary planned algorithm to JPEG compression. The encoded bits required to represent compressed image is less compared to JPEG. The PSNR values with proposed approach are greater than with the JPEG compression technique.

\section{ACKNOWLEDGMENTS}

Our sincere thanks to all the people who have helped us to complete this task. We are also very thankful to all the reviewers for their valuable ideas and suggestions.

\section{REFERENCES}

[1] Olivier Egger and Wei Li, "Very Low Bit Rate Image Coding Using Morphological Operators And Adaptive Decompositions" IEEE International Conference on Image Processing Vol-3, Nov-1994, PP No.326-330.

[2] Ricardo L. de Queiroz, Member IEEE, "Processing JPEG-Compressed Images and Documents" IEEE Transactions on Image Processing, Vol. 7, no. 12, december 1998, PPNo: 1661-1672. 
[3] Ravi Prakash, IEEE Member, Joan L. Mitchell, IEEE Fellow, and David A. Stepneski, "Enhanced JPEG Compression of Documents" IEEE International Conference on Image Processing Vol-3, Oct-2001, PP No: 494-497.

[4] Bai Xiangzhi, Zhou Fugen, "Edge Detection Based on Mathematical Morphology and Iterative Thresholding" IEEE International Conference on Image Processing Vol-2, Nov-2006, PP No: 1849-1852.

[5] Sreelekha G and P.S.Sathidevi, "An Improved JPEG Compression Scheme Using Human Visual System Model" IEEE, June 2007, PP No: 98-101.

[6] G.Srinivas, Prof Prasad Reddy P.V.G.D, and K.ramya "An N-Square Approach for Reduced Complexity NonBinary Encoding”, GJCST, PP No: 36-39, Vol: XI Issue XI.

[7] Ch. Ramesh, N.B.Venkateswarlu and J.V.R Murthy, "Filter Augmented JPEG Compressions" IJCA, Vol-60, No-17, Dec-2012. PP No: 1-5.
[8] Marlapalli Krishna, G.Srinivas and Prasad Reddy PVGD" Image Smoothening and Morphological operators Based JPEG Compression", JATIT, Vol.85, No.3, PPNo: 252-259, March 2016.

[9] Marlapalli Krishna, Prasad Reddy PVGD and G.Srinivas "A Smoothening based JPEG Compression for an Objective Image Quality Enhancement of Regular and Noisy Images, IJAER, Vol.11, No.6, PPNo:3799-3804, 2016.

[10] Utpal Nandi and Jyotsna Kumar Mandal “ Efficiency of Adaptive Fractal Image Compression with Archetype Classification and its Modifications", IJCA, Vol 38, 2016, Issue 2-3.

[11] G.Srinivas,P.Naga Srinivasu, T.Srinivasa rao and Ch.Ramesh "Harmonic and Contra Harmonic Mean Centric JPEG Compression for an Objective Image Quality Enhancement ofNoisy Images", Springer , International conference on smart computing and its application PP No143-152, 2017. 